Characterization of Collagen Type I and II Blended Hydrogels for

\title{
Articular Cartilage Tissue Engineering
}

Nelda Vázquez-Portalatín, ${ }^{1, \$}$ Claire E. Kilmer, ${ }^{2, \S}$ Alyssa Panitch, ${ }^{1}$ and Julie C. Liu ${ }^{1,2}$

${ }^{1}$ Weldon School of Biomedical Engineering, Purdue University, West Lafayette, IN 479072032, United States

${ }^{2}$ School of Chemical Engineering, Purdue University, West Lafayette, IN 47907-2100, United States

\section{Supplementary Information}

§These authors contributed equally to this work. 
Collagen Type II Incorporation Materials and Methods. Collagen type II in the gel was visualized using immunostaining with a primary antibody for collagen type II (Abcam 34712, Cambridge, MA). The gels were fixed in 3\% paraformaldehyde for 1 hour. After fixation, the gels were rinsed three times with $1 \times$ PBS, blocked with blocking buffer (5\% non-fat powdered milk in 1x PBS) for 1 hour at room temperature, and rinsed three times with $1 \times$ PBS. The gels were incubated with the primary antibody for 24 hours at $4^{\circ} \mathrm{C}$. The gels were rinsed with $1 \mathrm{x}$ PBS before being incubated with a goat anti-rabbit AlexaFluor® 555 (Cell Signaling Technology 4413S, Danvers, MA) for 24 hours at $4^{\circ} \mathrm{C}$. All confocal images were obtained using a $20 \mathrm{x}$ objective on a Nikon Ti-EC-1 Plus microscope (Nikon, Melville, NY). Nikon NIS-Elements AR software (version 3.2) was utilized to analyze fluorescence images.
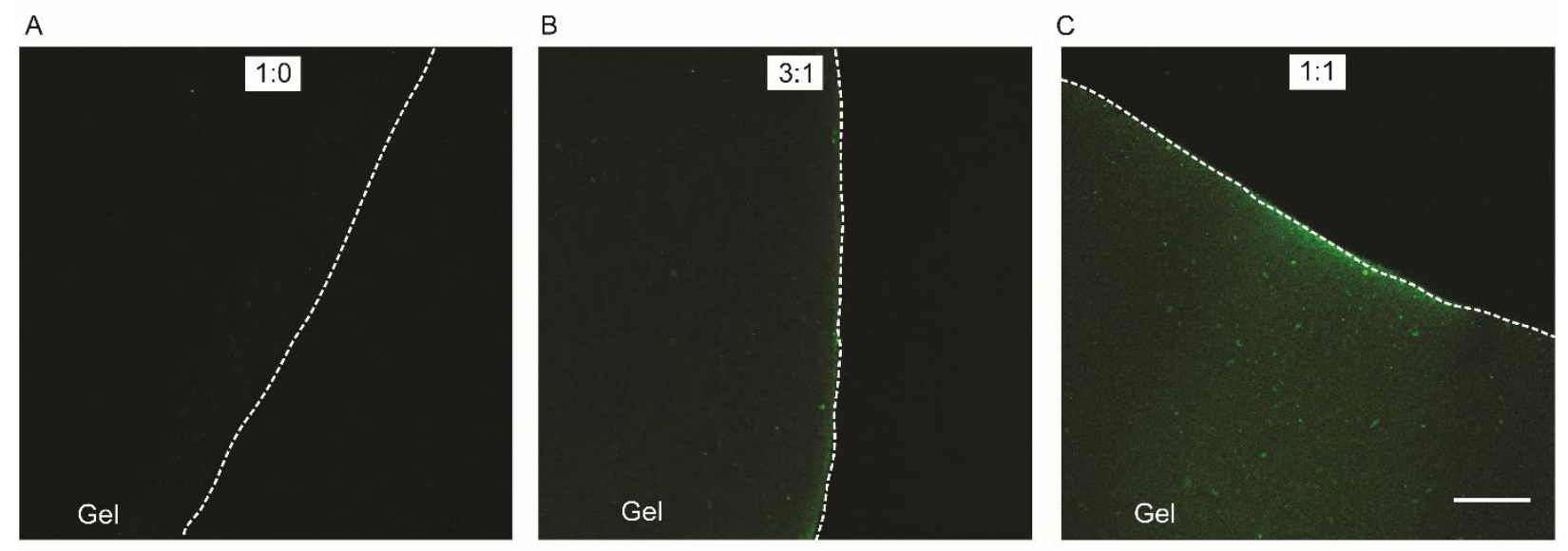

Figure S1. Representative images of immunostaining for collagen type II (green) hydrogels at different blends of (A) 1:0, (B) 3:1, and (C) 1:1 ratios of collagen type I to collagen type II. The boundary of the gel is indicated with the white, dashed lined, and the side containing the gel is notated in the figure. Scale bar represents $100 \mu \mathrm{m}$. 


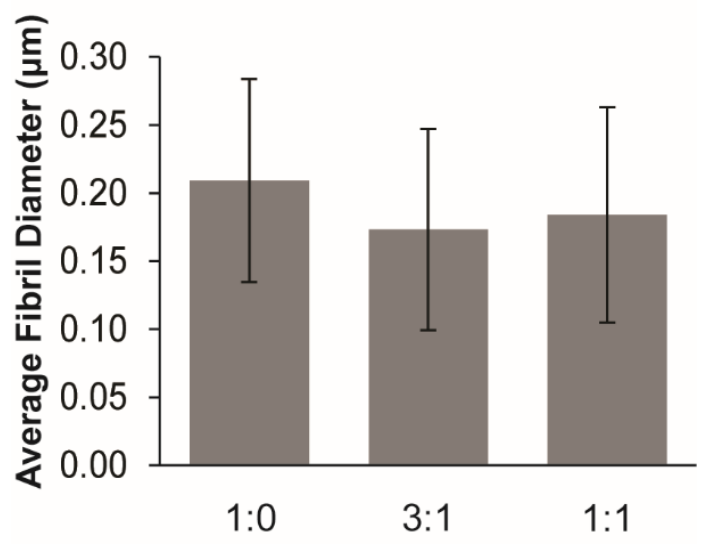

Figure S2. Average fibril diameter in the gels at different ratio blends. ANOVA and Tukey's post hoc tests were performed on the average fibril diameter data by using nested factorial models. For the average fibril diameter data, there were no significant differences observed between the different blends. Data $(n \geq 782)$ are represented as the mean \pm the standard deviation. 

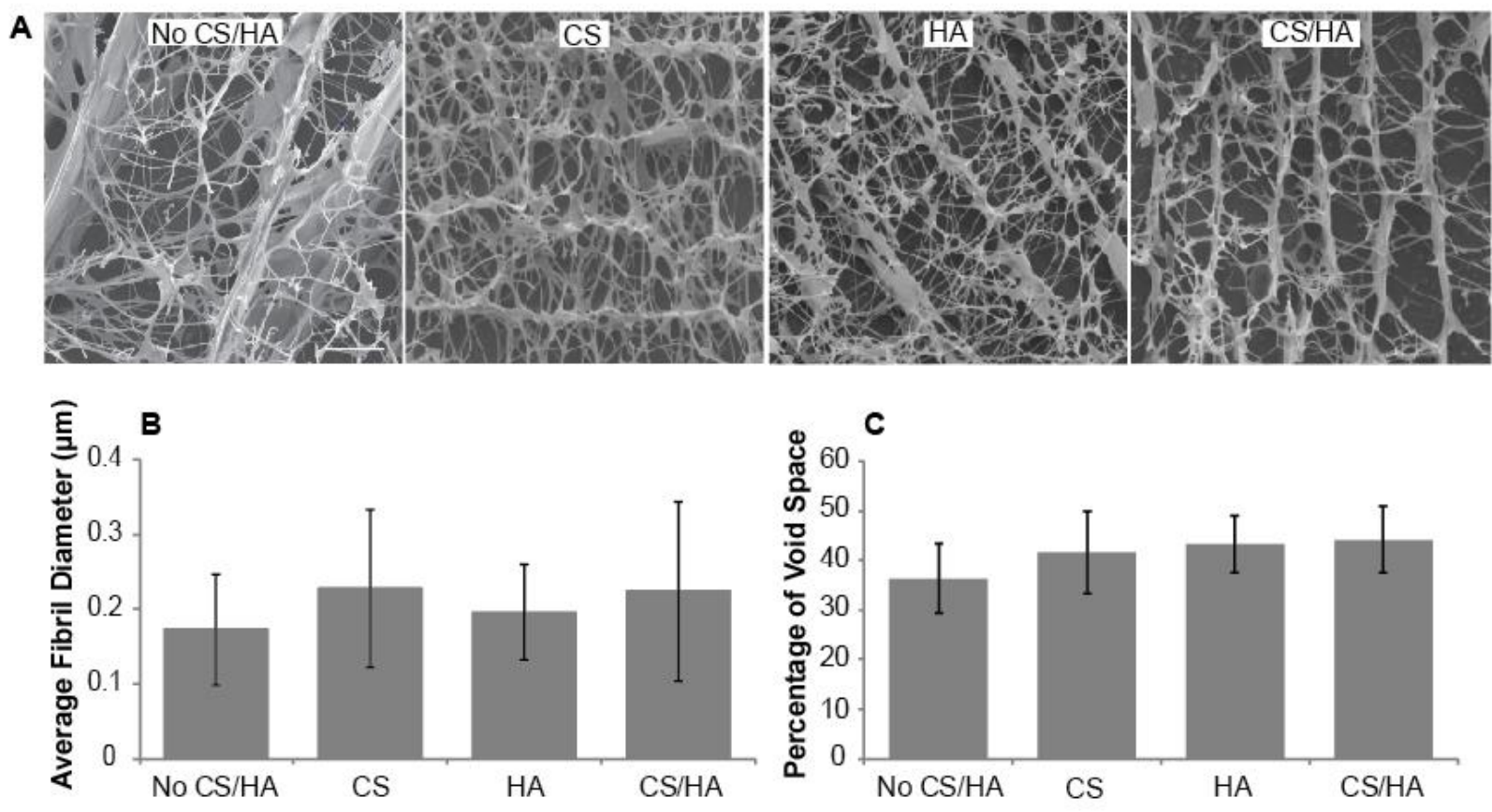

Figure S3. Effect of adding GAGs in collagen networks of different ratio blends. (A) Representative cryoSEM images of 3:1 collagen type I to collagen type II gels with no GAGs added or supplemented with CS, HA, or both CS and HA show the collagen fibril network within the gels. Scale bar represents $5 \mu \mathrm{m}$. (B) Average fibril diameter and (C) percentage of void space for the gels based on cryoSEM images obtained at 10,000x magnification. ANOVA and Tukey's post hoc tests were performed on the average fibril diameter and the percentage of void space data by using nested factorial models. There were no significant differences observed between gels that had no GAGs added or gels supplemented with CS, HA, or both CS and HA $(p>0.05)$. Data ( $n \geq 58$ for fibril diameter, $n \geq 8$ for void space percentage) are represented as the mean \pm the standard deviation. 


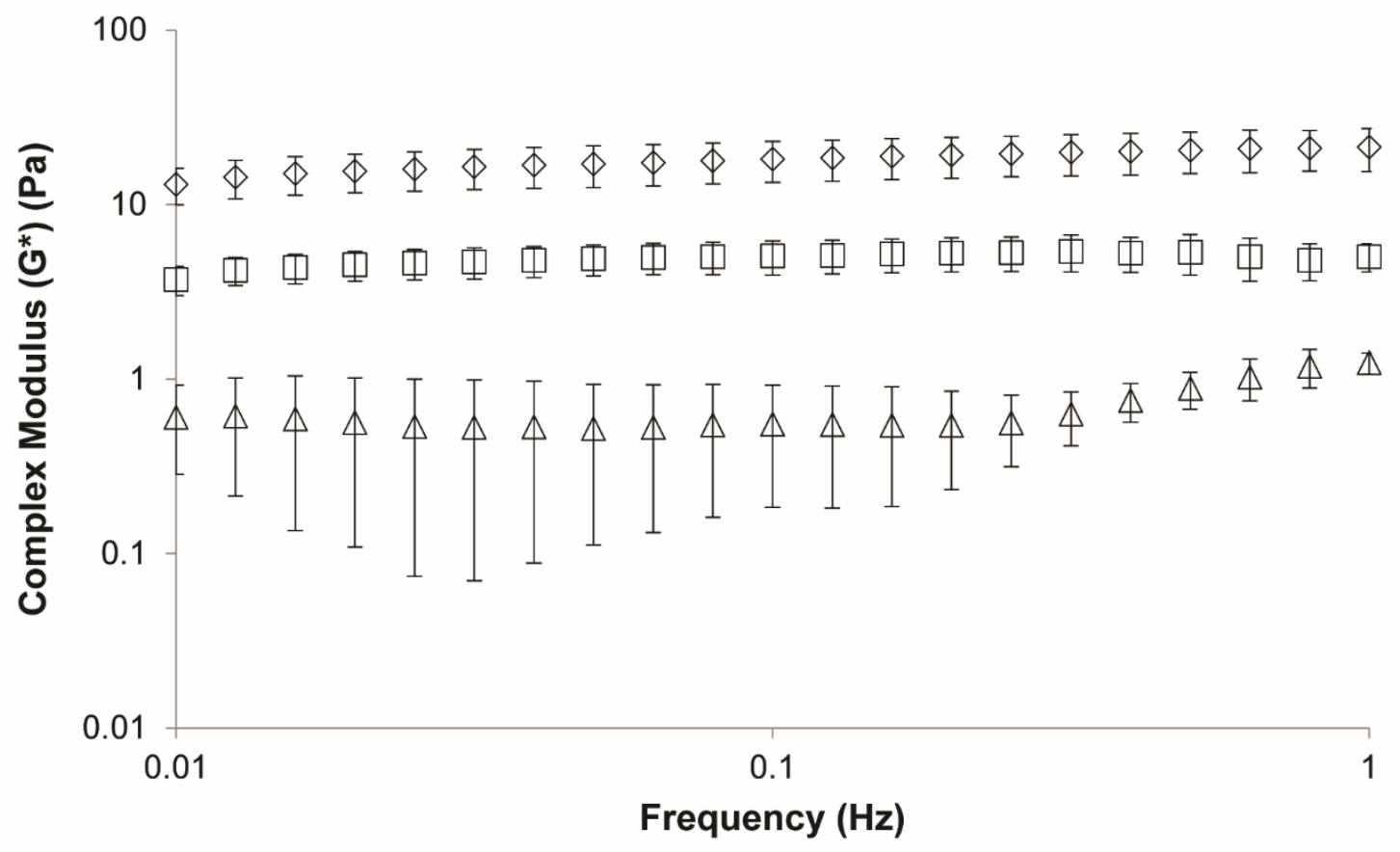

$\diamond 1: 0 \quad \square 3: 1 \quad \triangle 1: 1$

Figure S4. Complex modulus of gels prepared from mixtures of 1:0, 3:1, and 1:1 collagen type I to collagen type II. ANOVA and Tukey's post hoc tests were performed. At $1 \mathrm{~Hz}$, a significant difference $(p<0.05)$ was observed between the complex moduli for the three different ratios. Data $(n=4)$ are represented as the mean \pm the standard deviation. 